\title{
Are Comprehensive Quality Models Necessary for Evaluating Software Quality?
}

\author{
Klaus Lochmann \\ Technische Universität München \\ Software \& Systems Engineering \\ Munich, Germany \\ lochmann@in.tum.de
}

\author{
Jasmin Ramadani, Stefan Wagner \\ University of Stuttgart \\ Institute of Software Technology \\ Stuttgart, Germany \\ jasmin.ramadani, \\ stefan.wagner@informatik.uni- \\ stuttgart.de
}

\section{General Terms}

Experimentation

\section{Keywords}

Software quality, quality model, quality evaluation

\section{INTRODUCTION}

Software quality is a core concept of software engineering as we aim to build software systems in time, on budget, with suitable functionality and high quality. Almost any activity and method in software engineering influences the quality of software. To describe, evaluate and predict quality, we use quality models. They help us to understand what we need to pay attention to when building the system and also what to measure when we want to know the quality level of the system.

The fact that software quality is very complex and has many facets suggests that we also need comprehensive quality models to cover all these facets. Recent initiatives such as CISQ [26], Squale [20] and Quamoco [28] propose to some degree similar solutions in the form of large, operationalised quality models to support detailed measurement as well as the breadth of software quality. As a result, the quality models demand to collect a lot of data for various measures, some of them manually, with high effort or expensive tools. This, in turn, leads to comprehensive but also very large quality models. For example, the Quamoco base model contains 194 factors with an influence on quality measured by 526 measures for different programming languages.

\subsection{Problem Statement}

This comprehensiveness and level of detail of the comprehensive quality models have several advantages: They can help developers and quality engineers to better understand the meaning of measures and the relations between different factors with an impact on quality. Furthermore, they support the specification of detailed, testable quality requirements. Also for a comprehensible and precise quality evaluation, it is helpful to have all these details.

In contrast, there are many focused quality models, usually based on a small set of measures and regression or machine learning, to evaluate one or many quality aspects. They can be useful in practical settings where we prefer a less comprehensive and precise evaluation but at lower cost 
or effort. The various measures in the comprehensive models can come from diverse tools at differing costs and also for manual inspections with high effort. In software quality, however, a small, single defect can have a huge impact. Not including detectors for such defects can hence be a risk for the validity of the quality evaluation. So far, it is not clear what effects this trade-off between comprehensiveness and low application effort has. Even between the focused quality models, there are significant differences in the evaluation results [16].

\subsection{Research Objective}

Our overall aim is to make the application of quality models and their integration into the development process as simple and effortless as possible. To give immediate feedback to developers when quality problems have been introduced, we propose continuous quality control [8]. That ideally includes a continuous quality evaluation as well. Hence, our goal is to make the evaluation using a quality model fast and with low effort. In this paper, we concentrate on investigating the trade-off in using large, comprehensive quality models or focused quality models that use a limited set of measures for quality evaluation.

\subsection{Contribution}

Different quality models predict different measures as quality evaluation and, hence, are difficult to compare. Instead, we use an existing, comprehensive quality model and build focused quality models using various machine learning approaches based on the same set of measures that the comprehensive quality model uses. This way, we can analyse the accuracy of the prediction models. We use the maintainability part of the Quamoco base model as example for a comprehensive quality model, because its evaluations have a good correspondence to expert opinion [28], and established approaches, such as random forrest and regression, for building the focused models. In addition, we investigate the difference if manually collected measures are included. This is important, because those measures incur the highest costs to collect. We perform all the comparisons using an established approach: random partitions for model building and testing, comparison to a random predictor as baseline, the standardised accuracy measure SA [25] and effect sizes [6].

Our results show that it is possible to build a focused quality model based on a predictor: The best of the tested predictors relies only on 10 measures, compared to several hundred ones in the full model, but its accuracy is $61 \%$ with an effect size of 0.70 . Furthermore, our results show that the expert-based measures cannot be neglected, as the focused model built without expert-based measures works considerably worse for predicting evaluations which include expert-based measures.

\section{QUAMOCO QUALITY MODEL}

In the research project Quamoco, we developed a quality model for defining and evaluating quality [28]. The aim was that the resulting quality model should bridge the gap between abstract quality characteristics and concrete measures. Thus, the quality model defines factors, as an abstraction level between abstract quality characteristics and quantifiable measures. Such a comprehensive quality model support developers and quality engineers in different tasks. For instance, a Quamoco quality model can be used in re- quirements engineering as a checklist, so that one does not forget important quality characteristics and then add concrete factors and measurements so that the quality requirement is testable. The task a quality model can support best, however, is the evaluation of the quality of a software product.

We built the Quamoco base quality model as a collection of widely applicable quality factors and measures for the programming languages Java and C\#. We describe the concepts and structure of the base model, then its contents and, finally, the quality evaluation method based on the quality model.

\subsection{Concepts and Structure}

We started the Quamoco project, because we perceived a gap between abstract quality characteristics and concrete measurements [28]. We found it very hard to assign very detailed measures to the very broad quality characteristics, which are hard to further break down, and aggregate them to a sensible quality evaluation result. Therefore, we kept the abstract level, which we called the quality aspect level, and the concrete level which we called the measure level. As shown in Figure 1, we added an additional level, on which we describe factors of the product (properties of entities) that have an impact on the quality aspects and are measured by the measures. This is, for example, similar to the qualitycarrying properties of Dromey [10].

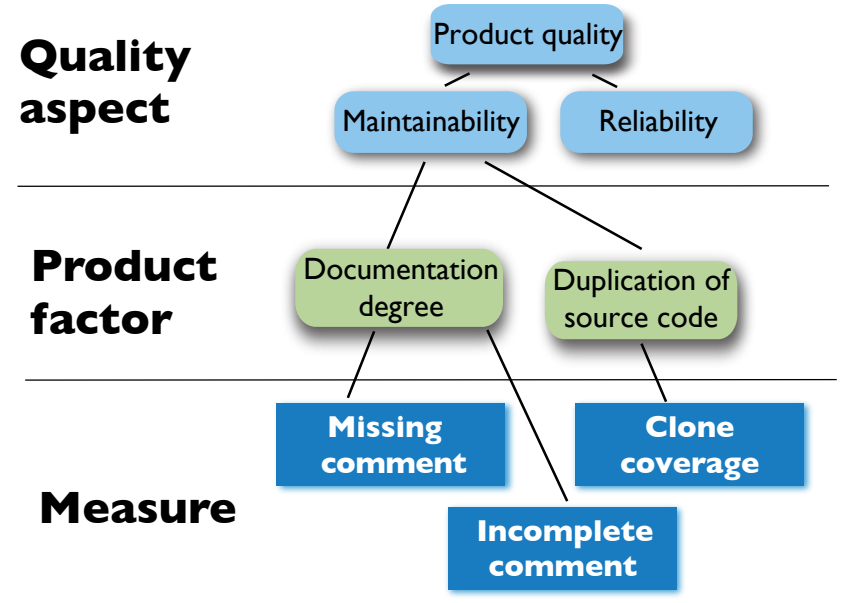

Figure 1: Schematic Structure of the Quamoco Base Quality Model

Quamoco quality models have more elements and details as shown in Figure 1. For instance, all the quality aspects, product factors and measures have IDs and descriptions. The impacts between product factors and quality aspects can be positive or negative and have a justification. There is additional information on how measurement results can be collected. We concentrate, however, on the information necessary for the following.

In principle, one can use any quality aspect hierarchy but to be consistent with others, we included the ISO/IEC 25010 product quality hierarchy in the Quamoco base quality model. The product factor level contains then various properties of the product, mostly concentrating on the source 
code. The documentation degree of Figure 1, for instance, has the following description:

A source code's documentation degree is high if it is commented as needed.

This product factor is not directly measurable, and thus we operationalize it by measures, which check whether comments are missing or incomplete. For missing comments, we have, for example, the tool ConQAT ${ }^{1}$ which gives us locations in Java source code where there should be a JavaDoc comment but it is not there. Missing comments are only part of the evaluation of documentation degree. It is also important what the comments contain. Therefore, there is also the manual measure incomplete comment which gives the proportion of incomplete comments in the source code. Together, the two measures can give a good impression of the degree of documentation of the source code.

To aid the structuring of product factors in a hierarchy and the clear definition of product factors, we associate an entity with each product factor. An entity is a part of the software product, e.g. the whole source code, sub-routines or statements. For documentation degree the entity is the comment in the source code.

We have done intensive empirical validations of the Quamoco approach and especially the Quamoco base model [28, 18]. We found that quality evaluations using the base model led to results that corresponded well with expert opinion. Especially the maintainability part of the base model seems to give very accurate evaluations. Therefore, we assume that the base model gives valid estimates of the quality level of a software system.

\subsection{Contents of the Quamoco Base Model}

The base model's main objective is to describe software quality in a way that a wide range of software products can be evaluated without requiring large effort upfront. To reach this goal, the Quamoco project partners conducted various workshops to collaboratively transfer their knowledge and experience into the Quamoco model structure.

The resulting quality model represents our consolidated view on the quality of software source code and is, in principle, applicable to any kind of software. It details quality down to particular analysis tools as instruments for the evaluation of Java and C\# systems, and, hence, enables comprehensive, tool-supported quality evaluation without requiring large adaptation or configuration effort.

In total, the base model comprises more than 1,500 model elements. Out of these, there are 201 product factors with an impact to quality aspects measured by 526 measures for both Java and C\#. This reflects that a product factor can be measured by more than one measure and it can also be relevant for several programming languages. The measures contain 8 manual ones and 518 that are provided by one of 12 different tools. The tools most relied upon are FindBugs (Java, 361 rules modelled) and Gendarme (C\#, 146 rules). Other tools integrated into our model include PMD and several clone detection, size and comment analyses, which are part of the quality assessment framework ConQAT.

To illustrate the contents of the base model, we will describe two examples of product factors together with their measures and impacts. As described above, the largest fraction of measures refers to static code analysis tools. One

\footnotetext{
${ }^{1}$ http://www. conqat.org
}

example is the FindBugs rule FE_TEST_IF_EQUAL_TO_NOT_A_NUMBER, which scans Java code for equality checks of floating point values with the Double.NaN constant. The Java language semantics defines that nothing ever equals $N a N$, not even $N a N$ itself, so that ( $x==$ Double.NaN) is always false. To check whether a value is not a number, the programmer has to call Double.isNaN(x). This rule is an instrument for the doomed test for equality to $\mathrm{NaN}$ measure, which measures the factor general expression applicability for comparison expressions, along with several other measures. This factor in turn impacts functional correctness, because the developer intended to check a number for $N a N$, but the actual code does not. It furthermore impacts analysability because to understand the intention of this construct demands additional effort.

Rule-based code analysis tools cannot detect every kind of quality problem. Therefore, the base model also contains product factors based on established research results, metrics, and best practices. For instance, identifiers have been found to be essential for the understandability of source code. Whether identifiers are used in a concise and consistent manner can only partly be assessed automatically [9]. Therefore, the factor conformity to naming convention for source code identifiers contains both automatic checks performed by several tools and manual measures for assessing whether identifiers are used in a consistent and meaningful way.

\subsection{Evaluation Method and Tooling}

We evaluate the quality of a software product using the quality model by adding evaluation specifications to product factors and quality aspects. For both, we evaluate on the scale $[0,1]$ expressing the degree to which the factor is present in the system. An evaluation of 0 means the factor is not present in the system, while 1 means the factor is fully present in the system. The evaluations of product factors are mappings from the values of its associated measures to the scale $[0,1]$. We define a minimum threshold and a maximum threshold; below the minimum threshold 0 is assigned and above the maximum threshold 1 is assigned. Between these thresholds, the evaluation changes linearly. The quality aspects evaluate simply by aggregating the product factors that have an impact on them. The aggregation is done by weighted sums similar to [1].

Our goal was to use very simple evaluations and aggregations so that it is easily comprehensible for practitioners. The challenge lay in defining reasonable thresholds for the evaluations. We calibrated these evaluations by measuring a large number of open source systems to find out what are "normal" values [17]. These defined the thresholds, we also reviewed and corrected based on expert opinion. For example, the measure clone coverage denotes the probability that for a randomly chosen statement in the source code, there exists a copy. We calculated the quartiles of the data from 125 Java systems, removed the outliers and used the minimum (0.0) and maximum (0.57) as thresholds.

The scale from 0 to 1 is very general and, hence, suitable to integrate various measures and factors. To help the quality engineer in interpreting the results for quality aspects, we added an additional step: the transformation into a grade scale. Being a German research project, we chose the German school grades as our grade scale. It goes from 1 to 6,1 being the best and 6 the worst grade. The analogy 
we used was a dictation at school. If you have more than $10 \%$ of your text wrong, you will get a 6 . Hence, $0-0.90$ gives a $6,0.90-0.92$ a 5 and so on. This turned out to be well comprehensible for practitioners [28].

For creating quality models and conducting automated quality evaluations, we developed a tool chain [7]. It consists of a graphical quality model editor used for quality modelling and the quality assessment toolkit ConQAT ${ }^{2}$ used for analysing artefacts and data. ConQAT and the Quamoco quality model editor are open source software licensed under the Apache 2.0 license.

ConQAT is a toolkit for the creation of quality dashboards that is configurable with a graphical domain-specific language. It provides diverse quality analyses out of the box and integrates with code analysis tools like Findbugs, PMD, or Gendarme. The results of the quality analyses are visualised in HTML dashboards. For conducting quality evaluations based on the quality model, the quality model editor allows to automatically generate a ConQAT configuration that contains all the required analyses to evaluate the software product according to the specifications in the quality model.

\section{RESEARCH STRATEGY}

We detail in the following how we conducted our experiment to compare the evaluations of the comprehensive Quamoco base model with a set of focused quality models built by machine learning.

\subsection{Research questions}

Our question overall is: Are comprehensive quality models necessary for evaluating software quality? Hence, we want to know if we can come to similar evaluation results using focused models, in our case built from existing data using machine learning approaches. In order to apply machine learning in a meaningful way, we need a large enough set of existing data. Thus, we will use the part of the Quamoco base model focusing on maintainability, because this part is the most comprehensive and well-elaborated one. It includes 378 maintainability-related measures and evaluates and aggregates them to a single grade for maintainability.

To further structure our study, we use two research questions reflecting the general performance of the focused models as well as the effects of expert-based measures:

- RQ 1: What is the performance of focused quality models built using machine learning algorithms?

The large number of measures in the Quamoco base model reflects the diversity of influences in the code onto maintenance. Under certain circumstances, however, we would like to be able to get an evaluation that is less accurate but needs less measures. For example, tools might not be available or analyses run too long for an hourly analysis. Therefore, we investigate to what extent it is possible to build a predictor, which calculates an evaluation result using a small number of measures.

- RQ 2: What is the performance of the focused quality models including additional expert-based measures?

An especially interesting use case for a focused quality model would be to avoid manual, expert-based measures. They are

\footnotetext{
${ }^{2}$ http://www. conqat.org/
}

usually collected during manual reviews, which means they are elaborate to collect and cannot be frequently measured. We build the predictors for RQ 1 using a large number of systems, for which only fully automated measures are available, however. For only 15 systems we have expert-based measures which are part of the Quamoco base quality model. In this research question, we focus on how the predictors perform for these 15 systems.

\subsection{Predictor models}

As focused quality models we use predictor models as known from machine learning, which predict the quality evaluation results of the comprehensive quality model. We use the following common machine learning algorithms to create the predictor models:

- Linear Regression: We use multiple linear regression, which is modelling the distribution of a dependent variable, using one or more independent variables. Linear regression models the relation between the dependent variable and the independent variables by fitting a linear equation to observed data. In our case, the independent variables are the measures of the Quamoco quality model, and the dependent variable is the evaluation result for maintainability. We do not use data transformation, as the inspection of the residual plots suggested a random distribution of the residuals and thus a good fit of the linear model.

- Classification Trees: We use the prediction method of classification trees according to [5]. This machine learning approach builds a tree in which the leafs represent the values of the dependent variable, the nodes represent branches based on the independent variables. Such a tree is constructed by recursively partitioning the data and deriving a branching condition for the resulting node. This approach is only applicable to categorical data, as in our case the school grades.

- Random Forests: Random Forests [4] are defined as a combination of tree predictors, whereby each tree is constructed based on a random selection of samples from the independent variables and Breiman's idea called bagging which reduces variance and helps to avoid overfitting. Using Random Forests we grow many classification trees where to classify an object the input has to be sent through all of the generated trees in the forest. Each of the trees performs classification and "votes" for the most popular class. At the end the forest is choosing the classification with the most votes by all of the trees in the forest.

Since not all predictors allow us to influence the number of variables they use, we apply a forward selection approach [12] for most predictors to incrementally increase the number of used variables. The forward selection is a simple and often used data-driven model building approach. In this approach, we start with an empty model containing no independent variables. The independent variables are added one at a time, testing all not yet included variables in each step and adding the most significant variable. The procedure is repeated until there are no more improvements in the model by adding another independent variable.

Concerning these machine learning algorithms, we use the 
following predictor models to investigate if they are useful for creating a focused quality model:

- Random Guessing: Instead of doing an actual predition based on independent variables, the random guessing approach returns a random value as predicted value. The distribution of the returned values is the same as that of the dependent variable. We use the random guessing approach as baseline to see how much the other predictors improve over it.

- 5\%-Quantile of the Random Guesses (5\% of the best guesses): This predictor looks at the $5 \%$ of the best guesses. It means that any value of accuracy that is better than this threshold has a chance of less than 1 in 20 to be a random occurrence.

- Linear Regression Model with Forward Selection: This predictor combines the linear regression and the forward selection algorithm described previously.

- Linear Regression Model with Backward Elimination: This predictor model applies the inversion of the forward selection to the linear regression. It starts with the maximum model consisting of all independent variables. The deletion of each variable is tested at each step and the variable that has the smallest significance is deleted.

- Linear Regression Model with Bidirectional Elimination: This predictor applies a combination of forward selection and backward elimination to the linear regression. At at each step, all independent variables are tested for either their inclusion or exclusion.

- Classification Tree with Different Complexity Parameters: When constructing classification trees it is possible to influence the number of used independent variables by predefining a required fit (denoted complexity parameter) of the independent variables with the dependent variable. The tree construction algorithm then selects the needed number of independent variables to achieve the required fit. We construct a series of classification trees with different complexity parameters and thus a different number of independent variables.

- Classification Tree with Forward Selection: Instead of controlling the number of used independend variables by the complexity parameter, for this predictor we apply the forward selection approach to classification trees.

- Random Forest with Forward Selection: Since the algorithm for constructing random forest predictors does not allows any control over the number of independent variables used, here we apply the forward selection approach to random forests.

\subsection{Model Comparison}

We need statistics for comparing the performance of predictors. A commonly used statistics for assessing the performance of a predictor is to calculate the magnitude of relative error (MMRE). Shepperd and MacDonell [25] state that "biased accuracy statistics such as MMRE are deprecated." They introduce a new validation framework to assess and compare prediction systems. We will use their framework and introduce the main statistics proposed by them in the following.

For measuring the performance of a predictor, we use the statistic mean absolute residual (MAR), defined as follows:

$$
M A R=\frac{\sum_{1}^{n}\left|\left(y_{i}-\hat{y}_{i}\right)\right|}{n}
$$

MAR is the mean of the absolute error values for the observations providing information with regard to the prediction model fit. It has the advantage of being an unbiased statistic, because it is not based on ratios.

To interpret the MAR statistic, Shepperd and MacDonnell introduce the idea of comparing a given predictor with a baseline predictor. As baseline predictor we take a simple approach of using the random guessing predictor. This results in the standardised accuracy measure (SA), which is the MAR relative to random guessing. For a suggested prediction technique $p_{i}$, where $i$ denotes a prediction technique used, the standard accuracy measure is defined as the following:

$$
S A_{p_{i}}=1-\frac{M A R_{p_{i}}}{\overline{M A R}_{p_{0}}}
$$

where $\overline{M A R}_{p_{0}}$ is the mean value of a large number of random guessing runs. It is defined as prediction of $\hat{y}_{i}$ for the target case $t$ by random sampling over all the remaining $n-1$ cases and takes $\hat{y}_{t}=y_{r}$ where $r$ is randomly chosen from $1 \ldots n \wedge r \neq t$. After many runs, the $\overline{M A R}_{p_{0}}$ converges on using the sample mean. The interpretation of SA is that the ratio shows how much $p_{i}$ is better than random guessing $p_{0}$. A value close to zero or a negative value is considered bad for the predictor model. A low MAR and a resulting SA close to $100 \%$ would mean that the predictor model is almost perfect.

In addition to the SA statistic, we use the effect size statistic to investigate how big the difference is really in relation to the overall variation in the random guesses. It is defined by Glass's $\Delta[25]$ as follows:

$$
\Delta=\frac{M A R_{p_{i}}-\overline{M A R}_{p_{0}}}{s_{p_{0}}}
$$

where $s_{p_{0}}$ is the sample standard deviation of random guessing. The Glass's $\Delta$ is a biased estimator for small sized samples or where there is a large difference in the size of the samples. It standardises the difference between two different prediction models and it gives context to the difference of amount of variations in the measures. The effect size as suggested by Cohen $[6]$ can be categorised as small $(\approx 0.2)$, medium $(\approx 0.5)$ or large $(\approx 0.8)$.

\subsection{Finding a Model with a Low Number of Measures}

Our goal is not to just find a predictor which performs well in predicting the quality evaluations of the comprehensive quality model, but to find a predictor which does so using a low number of measures. Thus, for each type of predictor, we construct a series of it, each using a different number of measures. For analysis and discussion, we then select two predictors for each type: First, we take a look at the predictor having the best result in terms of the SA statistic. Second, we select the predictor using the minimal number of measures, so that its MAR is not more than $10 \%$ 
less than the MAR of the best-performing predictor. Calculating percentages of the MAR statistic is valid since it is an absolute statistic.

\subsection{Study Objects}

For conducting automated quality evaluation, we need software products to be evaluated. We used the repository SDS [2, 23], containing about 18,000 open-source Java projects. These projects have mostly been retrieved from open source databases such as Sourceforge through a webcrawling approach. In essence, this repository contains mirrors of the version control repositories of the beforementioned databases. The SDS repository only contains the source code, not the binaries. For the quality evaluation, however, binaries compiled with the debug-option of the Java compiler are needed. We compiled all projects in a batch approach, because the effort to manually configure and compile them is prohibitive. The compilation of all 18.000 projects took about 30 hours, executed in parallel on 12 personal computers. Of all available projects about 6.000 compiled successfully. Others could not be compiled because of missing external libraries or because of code needed to be generated during the build process. We used all systems of the SDS repository larger than $5.000 \mathrm{LoC}$ as a benchmarking base, resulting in 2041 systems. We excluded smaller systems, because many open source repositories contain software projects initiated by single persons without finishing them; these projects then remain in the repository without ever being used $[3,24]$. Of the 2041 systems that compiled, the quality analysis tool run successfully on 1994 systems. Thus, these 1994 systems are used as study objects. The distribution of sizes shows that half of the systems are smaller than $11 \mathrm{kLoC}$ and $90 \%$ of the systems are smaller than $50 \mathrm{kLoC}$. Only 54 systems are larger than $100 \mathrm{kLoC}$, with the largest system having $477 \mathrm{kLoC}$.

Regarding RQ 2 we have 15 systems available, for which the expert-based measures of the quality model have been collected. Besides our own tool (ConQAT), we selected the most downloaded SourceForge ${ }^{3}$ projects written in Java. Of these projects we included only those, where it was possible to compile the source code on our own with reasonable effort (less than one hour work per system). The systems were: $\log 4 \mathrm{j}-1.2 .16$, jabref-2.3, tvbrowser-2.7.6, rssowl-2.0.6, checkstyle-5.3, apache-commons (of Dec 2010), conqatcore-2.6, conqat-bundles-2.6, fckeditor-2.6, freemind-0.8.1, hibernate-3.6.0, openproj-1.4, sweethome3d-3.0, thight-vncjava-1.3.10, and tomcat-6.0.24. For each of these systems, the expert-based measures were collected independently by two experts. The results were compared and differences in the expert judgement were discussed until a common judgement was achieved.

\subsection{Procedure}

The first step in our study is to collect the study data. For this purpose, we apply the Quamoco quality evaluation approach to the study objects using the Quamoco base quality model. As a result, for each study object, we get the measurement data for all measures of the quality model, as well as the aggregated values for the quality aspects. The full data is available in the PROMISE repository ${ }^{4}$. As we con-

\footnotetext{
${ }^{3}$ http://sourceforge.net/, accessed in December 2010.

${ }^{4}$ http://promisedata.googlecode.com
}

centrate on the maintainability part of the base model, we only use factors and measures related to maintainability.

In the next step, we create and evaluate the predictors for RQ 1. As independent variables all measures of the quality model are used, while as dependent variable, we use the evaluation of the quality aspect maintainability of the quality model. The experiments are implemented in a combination of Java and $\mathrm{R}^{5}$. $\mathrm{R}$ provides packages for all the predictors we use and it implements the statistics for evaluating the predictors. The Java program calls $\mathrm{R}$ scripts and is used to implement the forward/backward selection and the crossvalidation. We use a 4 -fold cross validation, which means the available study objects are partitioned into four subsets. Of the four subsets, one subset is used for validating the performance of the predictor, while the other three subsets are used for creating the predictor. This process is repeated four times, with each subset used as validation data once. As the result for the MAR statistic we use the average of the four runs. We also experimented with other folds (10-fold and 20 -fold cross-validation), but the results did not change significantly.

For RQ 2, the predictors are built using all study objects of RQ 1 . Since, for the study objects of RQ 1 the expertbased measures are not available, these predictors do not include the expert-based measures. These predictors are then evaluated by predicting for the 15 study objects of RQ 2 .

\section{RESULTS AND DISCUSSION}

In this section, we describe the results of the experiments and an interpretation according to the research questions of Section 3

\subsection{RQ 1: Performance of Focused Quality Models}

As outlined in Section 3, we built six predictors. We evaluated the performance of each predictor for varying numbers of variables used for prediction. Figure 2 shows the SA statistics of the predictors depending on the number of variables used by the predictor. All predictors perform better with more variables although there are also slight degradations for, e.g. classification tree with forward selection. Most predictors have an SA between $30 \%$ and $55 \%$ with below 10 variables, which is already a reasonable improvement to random guessing. The maximum SA is about $65 \%$ achieved by the random forest with forward selection.

According to the approach of Section 3 to compare the predictors, we determine the number of variables where each predictor performs best. Additionally, we determine the minimum number of variables, for which the performance cannot be improved by more than $10 \%$ by adding more variables. Table 1 gives the details. The minimal number of variables for the optimal MAR ranges between 16 and 87 . This seems to be due to slight changes in the MAR. If we look for a $10 \%$ improvement, the number of variables is reduced to $6-18$. This conforms also to our observation that in Figure 2 several of the predictors have achieved a close to maximum SA at about 10 variables.

Table 2 gives the MAR, SA and $\Delta$ statistics for the predictors with the number of variables determined above.

Regarding the performance of different predictors, in Figure 2 we see that the random forest predictor performs best

\footnotetext{
${ }^{5}$ http://www.r-project.org
} 


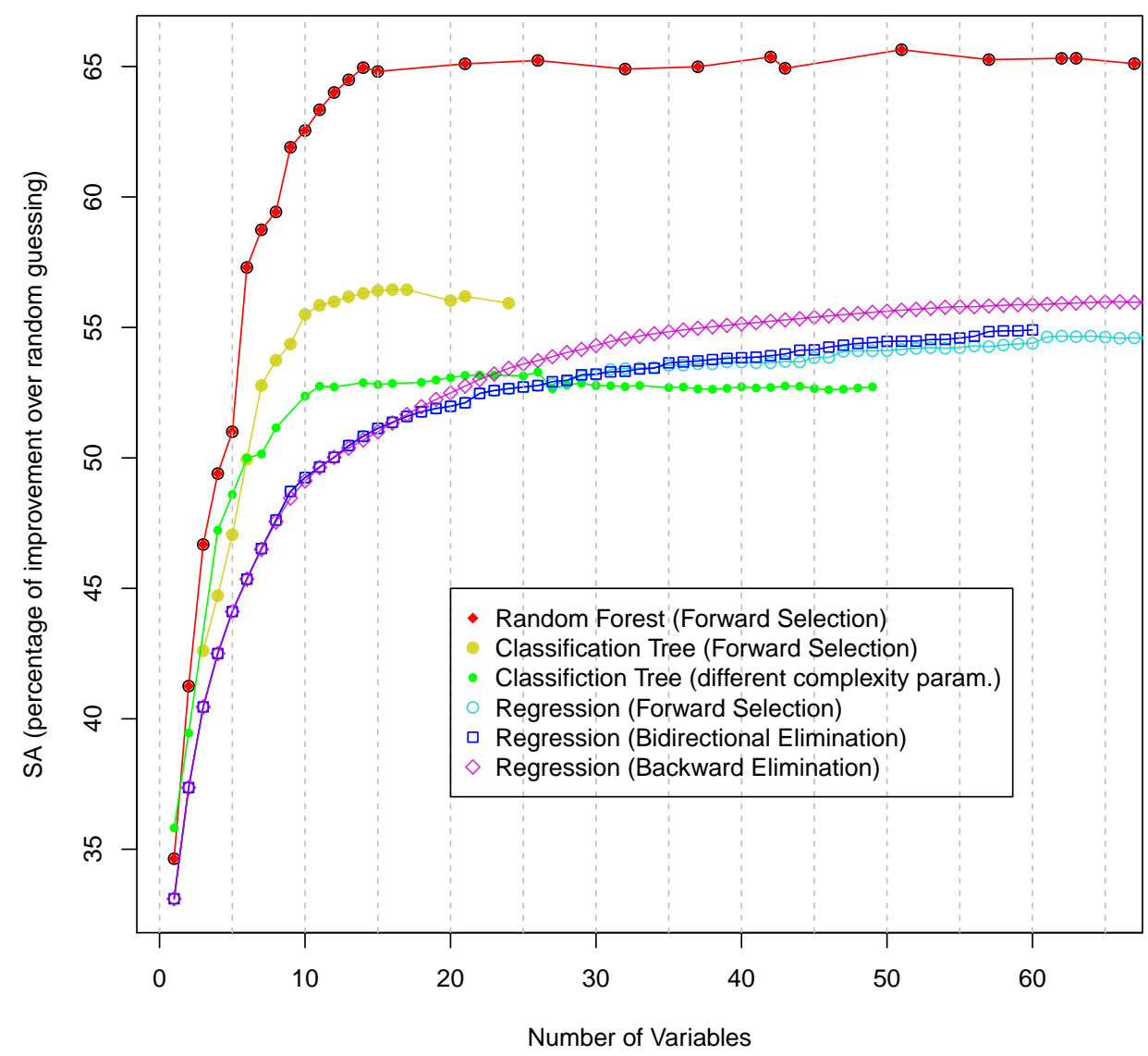

Figure 2: Performance of Predictors Depending on Numbers of Variables Used

\begin{tabular}{|l|c|c|}
\hline \multirow{2}{*}{ Predictor } & \multicolumn{2}{|c|}{ Number of Variables } \\
\cline { 2 - 3 } & $\begin{array}{c}\text { minimal } \\
\text { MAR }\end{array}$ & $\begin{array}{c}\text { 10\%-best } \\
\text { MAR }\end{array}$ \\
\hline Random Guessing & - & - \\
5\%-qtl. of Random Guess. & - & - \\
Linear Regr. (Forw. Sel.) & 62 & 13 \\
Linear Regr. (Backw. Elim.) & 60 & 13 \\
Linear Regr. (Bidir. Elim.) & 87 & 18 \\
Class. Tree (dif. cmplx. parm.) & 26 & 6 \\
Class. Tree (Forw. Sel.) & 16 & 7 \\
Random Forest (Forw. Sel.) & 51 & 10 \\
\hline
\end{tabular}

Table 1: Optimal Number of Variables for the Predictors

\begin{tabular}{|l|l|l|l|l|l|l|}
\hline \multirow{2}{*}{ Predictor } & \multicolumn{3}{|c|}{ minimal MAR } & \multicolumn{3}{c|}{$10 \%$-best MAR } \\
\cline { 2 - 7 } & MAR & SA & $\Delta$ & MAR & SA & $\Delta$ \\
\hline Random Guessing & 1.07 & $0.00 \%$ & & & & \\
$5 \%$-quantile of Random Guessing & 1.05 & $2.39 \%$ & & & & \\
Linear Regression (Forward Selection) & 0.49 & $54.67 \%$ & 0.61 & 0.53 & $50.47 \%$ & 0.57 \\
Linear Regression (Backward Elimination) & 0.48 & $54.90 \%$ & 0.62 & 0.53 & $50.47 \%$ & 0.57 \\
Linear Regression (Bidirectional Elim.) & 0.47 & $56.16 \%$ & 0.63 & 0.51 & $51.96 \%$ & 0.58 \\
Classification Tree (different complexity param.) & 0.50 & $53.29 \%$ & 0.60 & 0.53 & $50.15 \%$ & 0.56 \\
Classification Tree (Forward Selection) & 0.47 & $56.45 \%$ & 0.73 & 0.48 & $54.36 \%$ & 0.57 \\
Random Forest (Forward Selection) & 0.37 & $65.64 \%$ & 0.74 & 0.40 & $60.90 \%$ & 0.70 \\
\hline
\end{tabular}

Table 2: Statistics for Predictors 


\begin{tabular}{|l|l|l|l|l|l|l|}
\hline Number of Variables & 6 & 8 & 10 & 12 & 14 & 16 \\
\hline Data set without expert-based measures & $55.81 \%$ & $59.76 \%$ & $60.90 \%$ & $63.25 \%$ & $63.73 \%$ & $63.90 \%$ \\
Data set with expert-based measures & $32.11 \%$ & $33.75 \%$ & $35.27 \%$ & $43.16 \%$ & $42.51 \%$ & $46.72 \%$ \\
\hline
\end{tabular}

Table 3: SA statistic for data sets with/without expert-based measures

with some distance to the other predictors. A reasonably good prediction is achieved with 10 variables with the MAR statistic only decreasing by $10 \%$ over the best achievable MAR with 51 variables. The SA metric shows the random forest predictor with 10 variables is working $61 \%$ better than the baseline random guessing predictor. When using 51 variables it achieves an increase of $66 \%$ over the random guessing predictor. All other predictors achieve an SA value no better than $56 \%$ which is considerably lower than the value of the random forest predictor. Overall, we see a reasonable positive behaviour of the predictors.

The $\Delta$ statistic is about $\approx 0.6$ for all predictors, except for random forests and the classification tree with forward selection, which reach $\approx 0.75$. Considering the smaller, $10 \%$ models, only the random forest still achieves 0.70 . This is a medium to high effect size. Therefore, the reasonable improvement over random guessing has also a medium to high effect. Based on this, we conclude that a random forest predictor using 10 variables is well suited to be used as a focused quality model. Other predictors with roughly the same number of variables would still yield reasonable results.

\subsection{RQ 2: Performance Including Expert- Based Measures}

In the second research question, we used a random forest predictor, because it proved itself best in RQ 1 . The random forest predictor was built using all study objects of RQ 1, and therefore it does not include the expert-based measures. It was then used for predicting the quality assessment results for 15 systems, which included expert based measures.

Figure 3 and Table 3 show the SA statistic for different numbers of variables for two different predictors and data sets: (1) the data without expert-based measures is the result of the cross-validation predictor of RQ 1 (2) the data with expert-based measures is the result of the predictor especially built for RQ 2 and applied to the 15 systems mentioned above. The SA statistic for the prediction of the data including expert-based measures is considerably lower than in the results of RQ 1 . The SA statistic reaches its maximum of 48.60 with a random forest using 17 independent variables. Thus, the prediction performance is worse than that of the worst predictor in RQ 1 (when considering SA values for equal numbers of variables). Therefore, we conclude that the expert-based measures play an nonneglectible role. Accordingly, a predictor not considering these measures performs comparatively badly.

\section{THREATS TO VALIDITY}

Our investigation faces several threats to the validity of our results. We discuss internal and external validity threats as well as our mitigation strategies.

\subsection{Internal Validity}

One threat to internal validity is the choice and interpretation of the MAR, SA, and $\Delta$ statistics. For minimising

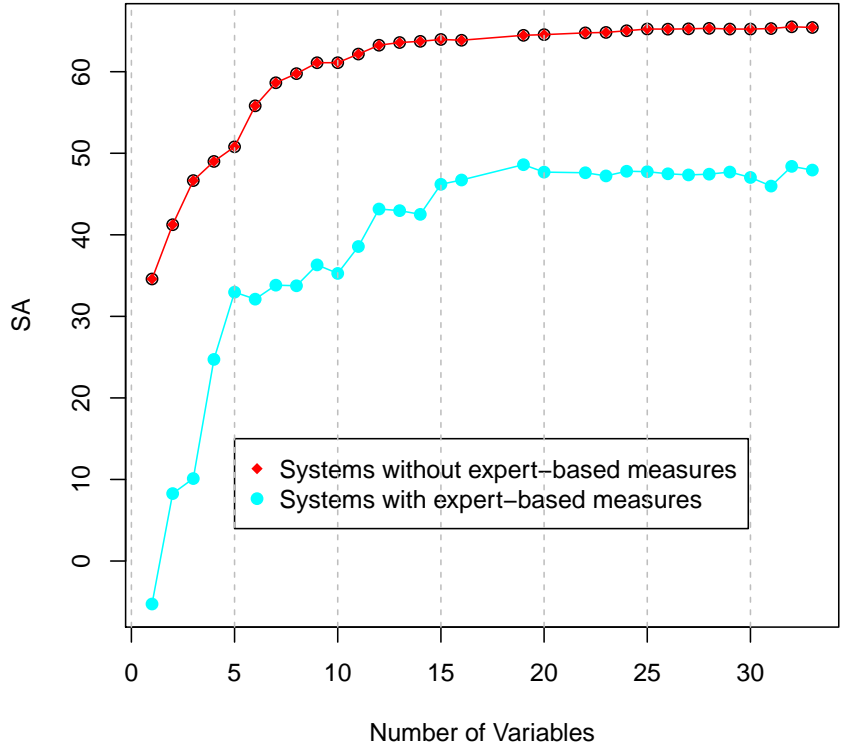

Figure 3: Random Forest Predictor used on data sets including and excluding expert-based measures

this threat, we aligned our research according to published guidelines as described in the study design.

In RQ 2 a threat to validity is the low number (15) of test systems. It could distort the resulting values for the SA metric. For assessing this threat, we conducted an additional experiment, to investigate the variance of the SA statistic when only 15 systems are used. We repeated the experiments of RQ 1 , but calculated the SA statistic by predicting the values of only 15 randomly chosen systems (1000 tries). The standard deviation of the SA statistic was 0.0795. Since, the values of SA were 0.6077 and 0.4586 respectively, we regard this threat as minor.

A third threat to internal validity is the set of predictors we tested. Maybe other prediction approaches (such as neural networks) could yield better results. However, since we regard the prediction performance of random forests as satisfactory this is no threat to our conclusions.

Finally, it is a threat to validity that in our experiments that showed correspondence with expert opinion [28], we included the manual measures but in our experiments for RQ 1 we could not include them as they are not available for so many systems. Hence, we cannot ensure that the Quamoco base model is really valid in that case. Therefore, we included RQ 2 to specifically look at the influence of manual measures.

\subsection{External Validity}

To generalise our results, the data and the systems we employed in our study need to be representative for a broader population. We made sure that our sample of open source 
systems contains a wide spectrum of systems with different sizes and different types of applications.

Despite the variations in the systems, they have all been built in Java. Several of the measures we used are specific for this programming language. Hence, the results may be different for other languages with other types of findings. As Java is a widely used programming language and in performing the study we did not observe something that seemed specific to Java, we expect this threat not to jeopardise the final conclusions.

We mostly employed automatic static analysis, because it is feasible for any company to collect data this way. Nevertheless, other types of analysis could give different results. We consider our restriction as necessary to be able to perform the study with this number of systems. Furthermore, it is comparable to the large amount of existing work on models based on static analysis data.

Finally, we used a quality model focusing on the quality aspect of maintainability. This could impair the generalisability to other quality aspects. However, other studies (cf. $[19,22]$ ) show that predicting security and defects based on static code analysis works well and, hence, we assume the same holds for maintainability.

\section{RELATED WORK}

In the research area of defect prediction, we can find a lot of work on source code metrics and their statistical analysis. Fenton et al. [11] and Hall et. al. [14] report on literature surveys summarising a large number of publications on defect prediction. Most of these studies have a different focus than our study. They build predictors for predicting faulty components within one system. We instead, build predictors being applied to a large number of software systems and predicting a given quality evaluation result instead of faultiness of components. Furthermore, these studies use general code metrics like complexity or lines-of-code as independent variables, while we use hundereds of static-code analysis rules. Nevertheless, some conclusions of these studies are relevant for our study.

Hall et. al. concludes that no general recommendation for a certain prediction technique can be given. Rather, depending on diverse context factors, different prediction techniques work better or worse. This supports our approach of using several prediction techniques and comparing the results.

To the best of our knowledge there is no study directly resembling our approach of using predictors to predict a quality evaluation result from a comprehensive quality model. The approach of Nagappan and Ball [21] comes closest to our approach, because they use the rules of static code checkers as independent variables, like we do. However, they again try to predict faulty components based on it with quite successful results. We take this result as an indicator that rulebased static code analysis is principally suited for quality analysis.

Another similar approach to ours is that of Wagner [27]. He described an approach to express a quality model similar to the Quamoco model using Bayesian nets. He found that the structure of the quality model transforms well into a Bayesian net but the prediction results were mixed. A reduction of measures was not considered.

While Hall et. al. generally do not recommend a certain prediction technique, there is some evidence, that in certain contexts random forests work best. For the NASA data set, both Guo et al. [13] and Lessmann et. al. [15] show a better performance of random forests over other prediction techniques. This result is in line with out results, where the random forest also worked best.

\section{CONCLUSIONS AND FUTURE WORK}

For evaluating the quality of software systems, comprehensive quality models have been built to reflect the diversity in software quality factors. The models rely on large numbers of measures (usually some hundreds) to get to a quality evaluation. On the other hand, there are focused quality models making use of a small set of measures for evaluating quality as well. Such a focused quality model has advantages as less effort is necessary for collecting data. If the inclusion of a high number of measures in comprehensive quality models really gives significantly different results than focused quality models has not been investigated so far.

Our results from comparing the Quamoco base model to focused models built using machine learning show that it is possible to build an evaluation model only relying on 10 measures while having a standardised accuracy of $61 \%$. The reduction from 378 measures in the comprehensive model to 10 measures facilitates the application in practice, because only 10 measures need to be calculated and, e.g., checked for false positives. However, if the evaluation model is to be used as a basis for contracting or design guidelines, it must be kept in mind that the results produced by the reduced model may differ from the results produced by the comprehensive model.

A major advantage of the focused model would be, if its reduced number of measures enabled to omit expert-based measurements. Our results indicate that the expert-based measures play a role that should not be neglected. The evaluation model built without expert-based measures works considerably worse for predicting assessments which include expert-based measures.

We put specific emphasis on the repeatability of our results. The used quality model and tool support for quality evaluation is freely available on the Quamoco web site ${ }^{6}$. We further publish our data and results in the PROMISE ${ }^{7}$ repository so that future research can easily reassess and improve our findings.

For future work we plan to investigate different constraints for selecting the measures used in the predictor. Possible constraints could be the number of different tools needed to calculate the measures, the price and effort for using a tool or the preciseness of measures.

\section{ACKNOWLEDGMENTS}

The presented work was partially funded by the German Federal Ministry of Education and Research (BMBF), grant "Quamoco, 01IS08023B".

\section{REFERENCES}

[1] T. Alves, C. Ypma, and J. Visser. Deriving metric thresholds from benchmark data. In Proc. International Conference on Software Maintenance (ICSM '10). IEEE Computer Society, 2010.

\footnotetext{
${ }^{6} \mathrm{http}: / /$ www. quamoco.de/

${ }^{7}$ http://promisedata.googlecode.com
} 
[2] S. Bajracharya, J. Ossher, and C. Lopes. Sourcerer: An internet-scale software repository. In Proc. Workshop on Search-Driven Development-Users, Infrastructure, Tools and Evaluation (SUITE '09). IEEE Computer Society, 2009.

[3] K. Beecher, A. Capiluppi, and C. Boldyreff. Identifying exogenous drivers and evolutionary stages in FLOSS projects. Journal of Systems and Software, 82(5):739-750, 2009.

[4] L. Breiman. Random Forests. Machine Learning, 45(1):5-32, 2001

[5] L. Breiman, J. Friedman, R. Olshen, and C. Stone. Classification and Regression Trees. Wadsworth, 1984.

[6] J. Cohen. A power primer. Psychological Bulletin, (112):155-159, 1992.

[7] F. Deissenboeck, L. Heinemann, M. Herrmannsdoerfer, K. Lochmann, and S. Wagner. The Quamoco tool chain for quality modeling and assessment. In Proc. International Conference on Software Engineering (ICSE '11). ACM Press, 2011.

[8] F. Deissenboeck, E. Juergens, B. Hummel, S. Wagner, Benedikt Mas y Parareda, and M. Pizka. Tool Support for Continuous Quality Control. IEEE Software, 25(5):60-67, 2008.

[9] F. Deissenboeck and M. Pizka. Concise and consistent naming. Software Quality Journal, 14(3):261-282, 2006.

[10] R. G. Dromey. A model for software product quality. IEEE Transactions on Software Engineering, 21(2):146-162, 1995.

[11] N. E. Fenton and M. Neil. A critique of software defect prediction models. IEEE Transactions on Software Engineering, 25(5):675-689, 1999.

[12] R. J. Freund and W. J. Wilson. Regression analysis: Statistical modeling of a response variable. Acad. Press, San Diego, Calif, 1998.

[13] L. Guo, Yan Ma, B. Cukic, and H. Singh. Robust prediction of fault-proneness by random forests. In International Symposium on Software Reliability Engineering (ISSRE 2004), pages 417-428. IEEE Computer Society, 2004.

[14] T. Hall, S. Beecham, D. Bowes, D. Gray, and S. Counsell. A Systematic Review of Fault Prediction Performance in Software Engineering. IEEE Transactions on Software Engineering, PP(99):1, 2011.

[15] S. Lessmann, B. Baesens, C. Mues, and S. Pietsch. Benchmarking Classification Models for Software Defect Prediction: A Proposed Framework and Novel Findings. IEEE Transactions on Software Engineering, 34(4):485-496, 2008.

[16] R. Lincke, T. Gutzmann, and W. Löwe. Software quality prediction models compared. In Proc. International Conference on Quality Software (QSIC 2010), pages 82-91. IEEE Computer Society, 2010 .

[17] K. Lochmann. A benchmarking-inspired approach to determine threshold values for metrics. In Proc. Workshop on Software Quality (WoSQ '12). ACM Press, 2012.

[18] K. Lochmann and L. Heinemann. Integrating quality models and static analysis for comprehensive quality assessment. In Proc. International Workshop on Emerging Trends in Software Metrics (WETSoM '11). ACM Press, 2011.

[19] T. Menzies, J. Greenwald, and A. Frank. Data mining static code attributes to learn defect predictors: Software engineering. IEEE Transactions on Software Engineering, 33(1):2-13, 2007.

[20] K. Mordal-Manet, F. Balmas, S. Denier, S. Ducasse, H. Wertz, J. Laval, F. Bellingard, and P. Vaillergues. The Squale model - a practice-based industrial quality model. In Proc. International Conference on Software Maintenance (ICSM '09), pages 531-534. IEEE Computer Society, 2009

[21] N. Nagappan and T. Ball. Static analysis tools as early indicators of pre-release defect density. In Proc. International Conference on Software Engineering (ICSE '05), pages 580-586. ACM Press, 2005.

[22] S. Neuhaus, T. Zimmermann, C. Holler, and A. Zeller. Predicting vulnerable software components. In Proc. Conference on Computer and Communications Security (CCS '07), CCS '07, pages 529-540, New York, NY, USA, 2007. ACM.

[23] J. Ossher, S. Bajracharya, E. Linstead, P. Baldi, and C. Lopes. SourcererDB: An aggregated repository of statically analyzed and cross-linked open source Java projects. In Proc. International Working Conference on Mining Software Repositories (MSR '09). IEEE Computer Society, 2009.

[24] A. Rainer and S. Gale. Evaluating the quality and quantity of data on open source software projects. In Proc. International Conference on Open Source Systems (ICOSS '05). UHRA, 2005.

[25] M. Shepperd and S. MacDonell. Evaluating prediction systems in software project estimation. Information and Software Technology, 54(8):820-827, 2012.

[26] R. M. Soley and B. Curtis. The consortium for IT software quality. In Proc. Software Engineering Approaches for Offshore and Outsourced Development (SEAFOOD 2010), volume 54 of LNBIP. Springer, 2010.

[27] S. Wagner. A Bayesian Network Approach to Assess and Predict Software Quality Using Activity-Based Quality Models. Information and Software Technology, 52(11):1230-1241, 2010.

[28] S. Wagner, K. Lochmann, L. Heinemann, M. Kläs, A. Trendowicz, R. Plösch, A. Seidl, A. Goeb, and J. Streit. The Quamoco product quality modelling and assessment approach. In Proc. International Conference on Software Engineering (ICSE '12). ACM Press, 2012. 